\title{
The 2020 update on anaphylaxis in paediatric population
}

\author{
Izabela Tarczoń ${ }^{1}$, Ewa Cichocka-Jarosz², Anna Knapp², Przemko Kwinta² \\ 'Przylądek Zdrowia, Krakow, Poland \\ ${ }^{2}$ Department of Paediatrics, Jagiellonian University Medical College, Krakow, Poland
}

Adv Dermatol Allergol 2022; XXXIX (1): 13-19

DOI: https://doi.org/10.5114/ada.2021.103327

\begin{abstract}
The objective of the review is to present recent updates on anaphylaxis in paediatric population worldwide. The article summarizes the results of epidemiological studies, diagnostic methods and treatments. We present a new WAO definition of anaphylaxis (2019), which broader criteria excluding dermal symptoms should facilitate faster lifesaving adrenaline use. Adrenaline remains the best treatment to manage severe symptoms and to prevent biphasic reactions. There is ongoing effort to increase adrenaline use, such as modified autoinjectors, individual training, and diversified dosing. There are five independent risk factors of lethal anaphylaxis in children, including history of asthma, almost immediate onset of symptoms, unwell appearance, tachycardia and hypotension. We also report improvements in diagnostics, like component-resolved diagnostics, and novel therapies stimulating immunotolerance. We signal the development of ICD-11 with updated coding of anaphylaxis, which corresponds better to clinical observations.
\end{abstract}

Key words: anaphylaxis, definition, children, triggers, phenotype, intervention, prevention.

\section{Introduction}

The goal of our work is to present a traditional review of recent publications on anaphylaxis in children, with a particular emphasis on changes in the newest recommendations on its diagnosis, novel diagnostic methods, verification of adrenaline-based standard treatment, and upcoming advances in prevention approaches. The reviewed and summarized information is to provide an update of practical guidelines for the medical personnel to improve the care of paediatric patients.

\section{Definition and diagnosis}

Anaphylaxis is the most severe form of an allergic reaction. Its diagnosis is purely clinical. The characteristic symptoms, which affect dermal, respiratory, cardiovascular or gastrointestinal systems, develop from minutes to a few hours after exposure to a known or potential trigger. There were several published anaphylaxis definitions [1-5], which share a common denominator of anaphylaxis life-threatening potential. The clinical criteria of anaphylaxis were first proposed at the second NIAID/ FAAN Symposium in 2005 [1] (Table 1). Subsequently they were adopted by WAO [5] and EAACI [6] and have been used since then. They identify anaphylaxis with $95 \%$ sensitivity (95\% Cl: $85-99)$, but only $71 \%$ specificity $(95 \% \mathrm{Cl}$ :
61-79), the positive predictive value equals 64\% (95\% Cl: 53-73), while the negative predictive value equals $96 \%$ (95\% Cl: 89-99) [7]. Usefulness of the above criteria was the topic of a decade-long debate regarding their role in correct diagnosis and proper use of adrenaline. Therefore, the WAO Anaphylaxis Committee proposed a revised definition and clinical criteria of anaphylaxis in 2019 [8]. Accordingly, anaphylaxis is recognized as a serious systemic hypersensitivity reaction with usually rapid onset and potentially life-threatening compromise of breathing and/or the circulation, which may occur without typical skin features. New clinical criteria without previously obligatory dermal symptoms appear to facilitate the diagnosis of anaphylaxis and expedite introduction of adequate intervention. Currently there are two scenarios of anaphylaxis, which may present as 1) typical dermal features AND other significant symptoms from at least one other system OR 2) disorders of the respiratory system and/or cardiovascular system following exposure to a known or potential allergen (Table 1). The revised definition of anaphylaxis is included in an updated version (ICD-11) of the International Statistical Classification of Diseases and Related Health problems (ICD) of the World Health Organization (WHO) [9], which will become effective starting 1 January 2022 as indicated at the WHO webpage (http://www.who.int/classifications/icd/en/, retrieved 27 December 2020).

Address for correspondence: Assoc. Prof. Ewa Cichocka-Jarosz, MD, PhD, Department of Paediatrics, Jagiellonian University Medical College, 265 Wielicka St, 30-663 Krakow, Poland, phone: +48 1233394 76, e-mail: ewa.cichocka-jarosz@uj.edu.pl Received: 28.12.2020, accepted: 15.01.2021. 
Table 1. Clinical criteria for the diagnosis of anaphylaxis NIAID/FAAN of 2005 and WAO of 2019

Second National Institute of Allergy and Infectious Diseases/Food
Allergy and Anaphylaxis Network (NIAID/FAAN) symposium, 2005

\section{[Sampson JACI, 2006; 391-7]}

One of the three following criteria is fulfilled:

1. Acute onset of an illness (minutes to several hours) with involvement of the skin, mucosal tissue or both (e.g. generalized hives, itching or flushing, swollen lips-tongue-uvula)

AND AT LEAST ONE OF THE FOLLOWING

a. Respiratory disorder (e.g. dyspnoea, wheeze, bronchospasm, stridor, decreased PEF value, hypoxemia)

b. Reduced blood pressure or symptoms of end-organ dysfunction (hypotonia [collapse], syncope, urinary incontinence)

2. Two or more of the following situations that occur suddenly after exposure to a likely allergen for that patient (minutes to several hours):

a. Involvement of the skin, mucosal tissue or both (e.g. generalized hives, itching or flushing, swollen lips-tongue-uvula)

b. Respiratory disorder (e.g. dyspnoea, wheeze, bronchospasm, stridor, decreased PEF value, hypoxemia)

c. Reduced blood pressure or symptoms of end-organ dysfunction (hypotonia [collapse], syncope, urinary incontinence)

d. Persistent gastrointestinal symptoms (e.g. crampy abdominal pain, vomiting)

3. Reduced blood pressure after exposure to a likely allergen for that patient (minutes to several hours):

a. Infants and children: low systolic blood pressure (age specific) or greater than $30 \%$ of decrease in systolic BP

b. Adults: systolic BP of less than $90 \mathrm{~mm} \mathrm{Hg}$ or greater than $30 \%$ decrease from that person's baseline

1. Low systolic blood pressure - a decrease in systolic blood pressure greater than $30 \%$ from that person's baseline or in infants and children under age of 10 : systolic BP less than ( $70 \mathrm{~mm} \mathrm{Hg}+[2 \times$ age in years] or in older children or adults - systolic blood pressure less than $90 \mathrm{~mm} \mathrm{Hg}$.

2. Laryngeal symptoms comprise: stridor, voice change, odynophagia.

3. Allergen is a substance (usually protein) capable of triggering immunological response, which may lead to an allergic reaction. Most allergens act by route which lgE is the mediator for, but some non-allergen triggers may act independently of IgE (for instance by a direct mast cell activation).

4. Most allergic reactions occur within 1-2 h from exposure, usually much faster. Reactions can be delayed in case of some food allergens (e.g. alpha-gal) or in the context of immunotherapy, up to $10 \mathrm{~h}$ following exposure.

\section{Epidemiology}

The true global extent of anaphylaxis is hard to estimate [10]. In the European population, data have been mainly based on the European Anaphylaxis Registry (EAR) since 2014 [11]. The Registry currently contains 14706 cases, including 4256 of children and adolescents (The Anaphylaxis Registry ANAPHYLAXIE.net, https:// www.anaphylaxie.net/en/\#tabs-quote-1552943354-58-1, retrieved 22 December 2020).

The previous data analyses originating from the EAR included a study of risk factors of biphasic anaphylaxis and epinephrine administration [12], phenotype and risk factors of venom-induced anaphylaxis (VIA) [13], anaphylaxis in children and adolescents [14], in the elderly [15], and a summary of preventive measures to avoid anaphylaxis [16]. A systematic review of the literature on the epidemiology of anaphylaxis in Europe shows the incidence rate between 1.5 and 7.9 in 100,000 person-years, and the lifetime prevalence of $0.3 \%(95 \% \mathrm{Cl}: 0.1-0.5)$ [3].
One of the two following criteria is fulfilled:

1. Acute onset of an illness (minutes to several hours) with involvement of the skin, mucosal tissue or both (e.g. generalized hives, itching or flushing, swollen lips-tongue- uvula)

AND AT LEAST ONE OF THE FOLLOWING

a. Respiratory disorder (e.g. dyspnoea, wheeze, bronchospasm, stridor, decreased PEF value, hypoxemia)

b. Reduced blood pressure or symptoms of end-organ dysfunction (hypotonia [collapse], syncope, urinary incontinence)

c. Severe gastrointestinal symptoms (e.g. acute crampy abdominal pain, recurrent vomiting), especially after exposure to the allergen other than food)

2. Acute onset of hypotension ${ }^{1}$ or bronchospasm or laryngeal edema ${ }^{2}$ right after exposure to a known or a highly probable allergen $^{3}$ for that patient (minutes to several hours ${ }^{4}$ ), even with absence of typical involvement of the skin
The American College of Allergy, Asthma and Immunology estimates the general incidence of anaphylaxis in the USA from 30-60 cases to 950 cases in 100,000 personyears with lifetime prevalence of $0.05-2.0 \%$ [4]. Anaphylaxis accounts for up to $0.26 \%$ of all hospital admissions [17]. The death rate remains constant at $0.5 \%$ to $1 \%$. This is in contrast to Australia [18], where the number of fatal anaphylaxis cases due to all causes rose annually by $6.2 \%$ from 1997 to 2013, mainly because of adverse food reactions [18]. The latest published data on prevalence of anaphylaxis in Poland are based on the National Health Fund's records for 2008-2015 and a 2015 questionnaire-based survey collected from 305 allergists [19]. In 2015, 3144 people received treatment for anaphylactic shock with an estimated prevalence rate of anaphylaxis equal to 8.2 per 100,000 with the highest prevalence rate in women aged $50-54$ years ( 14.5 per 100,000$)$. The most common causes of anaphylaxis included insect venom (41.4\%), food (29.8\%) and drugs (17.4\%) [19]. 


\section{Epidemiology in children}

In children, the combined data from 29 studies indicated the anaphylaxis incidence rate between 1 and 761 per 100,000 person-years. The incidence rates varied in different continents: 2.3-761 per 100,000 person-years in Europe, 0.8-70 per 100,000 person-years in North America. Globally, prevalence estimates for anaphylaxis in the paediatric population ranged from $0.04 \%$ to $1.8 \%$ [20].

In childhood until the age of 10 , anaphylaxis occurs more often in boys than girls [21], probably because of the higher incidence of food allergy in the former [22]. In the prepubescent age, the rates of both severity [23] and fatality [24] are comparable in both genders. Over 13 years of age, there is a gradual increase in the incidence of severe anaphylaxis in males, in particular in venom [25] or drug-induced anaphylaxis [26].

The majority of the new cases is diagnosed in children under the age of 4 [3]. Overall, the highest incidence rate is for the food-induced anaphylaxis (1.4-76.7 per 100,000 person-years with the prevalence estimates between 0.3 and $1.2 \%$ [20]. The hospitalization rate due to anaphylaxis among infants and toddlers was constant between 2006 and 2015 in the United States. However, it increased significantly in the older children (age $>3$ $<18$ years) [27]. The incidence rate of anaphylaxis induced by a specific food allergen (peanuts, nuts, fruit, milk, seafood, eggs) in children was between 0.1 and 9.7 per 100,000 person-years, which was higher than the one for venom, anaesthesia or serum triggers [20].

Even though the highest rate of food-induced anaphylaxis was observed in the youngest age group, the latest epidemiological data from the United States [28], Italy [29], and Australia [30] showed an increased number of hospitalized older children due to food-induced anaphylaxis, especially among the 13-17-year-olds. It is especially worrisome since the estimated risk of morbidity and mortality due to food-induced anaphylaxis is greater in the adolescents compared to other age groups [21].

\section{Triggers}

Worldwide, the most common triggers of anaphylaxis are food, drugs and venom, though geographic differences exist due to nutritional habits typical in the particular region [31]. In the European paediatric population foods (66\%) predominate, then venoms (19\%) and drugs (5\%) as demonstrated by the EAR between 2007 and 2015 [14].

The type of triggers changes with age. Foods are the most common allergens in children under 6 years, with milk and hen egg white prevailing in the infants younger than 2 years. Anaphylactic reactions to hazelnuts and cashews are typical for the pre-school group, while peanuts trigger anaphylaxis in populations of any age. According to EAR, venoms (wasps more often than bees) are the leading cause of anaphylaxis in school-age children and teenagers, whereas medications (analgesics and antibiotics) result in anaphylaxis mostly in the latter age group [14]. The most common factors that necessitate hospital readmissions due to anaphylaxis include food allergens, such as seeds and tree nuts, age groups of 6-12 and 13-17, and weekend days [32].

The fatal anaphylaxis in children is most often caused by drugs, foods and venoms [21, 22, 30]. The common food triggers include peanuts and tree nuts, which predominate in the general paediatric population [25], and cow milk typical for the youngest children [22]. Some food triggers inducing fatal anaphylaxis are region specific, such as seafood in Australia [30].

Another difficult issue is idiopathic anaphylaxis (IA) for which a trigger cannot be identified. It is less common in children compared to the adult population. The Network for Online Registration of Anaphylaxis (NORA) in Europe reports a surprisingly low number of IA cases (6.5\%), but the data collection might have been somehow affected by the particular profile of that network highly specialized in allergy diagnosis [11]. Contrary to NORA results, in the review of 40 paediatric anaphylactic cases treated in an emergency department (ED) in the United States in the year 2014, 17.5\% of cases were classified as IA [33]. Evaluation of the potential triggers in idiopathic anaphylaxis has improved due to introduction of a component-resolved diagnostics of hidden allergens found in food ingredients such as meal flavours (e.g. celery, mustard, herbs, spices, fenugreek in particular, legumes - especially lupin), food additives (natural food colourings such as carmine, or preservative sodium metabisulphite, which is present in white and rose wine or light coloured fruit juices), foods eaten for health reasons (quinoa, buckwheat, millet, amaranth) or alpha-gal substances (delayed reactions after consumption of red meat) [34]. CRD allows to determine causative agents in anaphylaxis cases which previously were considered idiopathic even in $1 / 5$ of them [35].

\section{Phenotypes}

The symptoms of anaphylaxis may vary in different patients, and even in subsequent episodes in the same person. Phenotypes of anaphylaxis, similarly to phenotypes of asthma, may alter according to its trigger. Typically, food allergy is associated with vomiting, nausea and gastrointestinal symptoms [36], while allergy to drugs and insect venom results in hypotension regardless of age [13, 37].

A report based on the Beijing Pharmacovigilance Database (2004-2014) showed that children of 0-5 years were more likely to develop cyanosis than children of 13-17 years $(\mathrm{OR}=5.14,95 \% \mathrm{Cl}: 1.74-15.20)$, children of 13-17 years were more likely to develop hypotension than children of $6-12$ years $(\mathrm{OR}=11.79,95 \% \mathrm{Cl}: 2.28-60.87)$, and to manifest both neurological symptoms $(\mathrm{OR}=3.56$, 95\% Cl: 1.26-10.08) and severe anaphylaxis com- 
pared to the children of $0-5$ years $(\mathrm{OR}=15.46,95 \% \mathrm{Cl}$ : 1.85-129.33) [38]. The EAR case control study of the VIA ( $n=3,612$, including $20 \%$ of patients younger than 22 years $)$ and non-VIA $(n=3,605)$ patients matched for age and gender demonstrated that hypotension was a predominant manifestation in any age VIA group when compared to either other non-VIA triggers or symptoms of different body systems [13]. There were episodic incidents of drug-induced perioperative anaphylaxis reported in children, which compromised a total of 29 cases from the UK, USA and France (average age: 11 years) during a 10-year period [39]. The severe symptoms occurred in $86 \%$ of the group, and $14 \%$ developed circulatory failure. The first symptom was hypotension (59\%), then tachycardia and bronchospasm [39].

Another study based on the data from the Paediatric Research Consortium reported 54 cases of drug-triggered reactions (incidence 1 : 4219) out of 227,833 allergic cases [40]. There were 6 cases consistent with anaphylaxis diagnosis (incidence 1 : 37972). The following sedative and/or analgesic medications showed a significant association with development of allergic reactions: midazolam $(\mathrm{OR}=2.2 ; 95 \% \mathrm{Cl}: 1.2-3.9)$, ketamine $(\mathrm{OR}=3.8$; 95\% Cl: 2.1-7.1), methohexital (OR = 48.8, 95\% Cl: $14.9-$ 159.9), and morphine (OR $=4.4 ; 95 \% \mathrm{Cl}: 1.04-18.2)$ [40]. There were no mortalities. The most recently described troubling phenomenon is a potential transfer of atopy and an associated risk of anaphylaxis during transplant surgeries [41].

\section{Risk factors}

There is a number of recent reports on the risk factors of severe anaphylaxis. A Spanish study defined severe anaphylaxis as a clinical condition that fulfilled one or more of the following criteria: administration of two or more doses of epinephrine, clinically important biphasic reaction, endotracheal intubation, intensive care unit admission, and/or death. Out of 453 episodes of anaphylaxis, 61 were classified as severe anaphylaxis (13.5\%, 95\% Cl: 10.6-16.9): 53 patients (11.7\%) required more than one dose of epinephrine, 14 (3.1\%) developed clinically important biphasic reactions, there were $2(0.4 \%)$ intubations in the ED, and 6 (1.3\%) admissions to the intensive care unit, with no fatalities reported. The identified independent risk factors for severe anaphylaxis included history of asthma (OR $=2.70,95 \% \mathrm{Cl}: 1.43-5.11)$, rapid onset of symptoms less than 5 min after the allergen exposure $(\mathrm{OR}=2.62,95 \% \mathrm{Cl}: 1.41-4.87)$, unwell appearance $(\mathrm{OR}=2.97,95 \% \mathrm{Cl}: 1.38-6.40)$, tachycardia $(\mathrm{OR}=2.34,95 \% \mathrm{Cl}: 1.19-4.96)$, and hypotension (OR = 3.72, 95\% Cl: 1.09-12.76) [42]. Yet another study did not confirm the role of asthma in severity of anaphylaxis [43], defined by a specific clinical presentation or need for therapy of respiratory failure. The presented multivariate analyses showed that children with a history of asthma were not more likely to have severe anaphylactic reactions (OR $=0.97 ; 95 \% \mathrm{Cl}$ : 0.67-1.39) [43].

\section{Biomarkers}

Anaphylaxis remains a clinical diagnosis, and biomarkers have no role in acute management. However, they are crucial in the specialist allergy evaluation to confirm the diagnosis and support differential diagnosis. Tryptase is the most useful one, also in the post-mortem evaluation $[44,45]$. Even though an elevated tryptase level has a high (93\%) positive predictive value, its negative predictive value is only $17 \%$ [46]. It is best to collect a blood sample 1-2 $\mathrm{h}$ from the onset of symptoms, and freeze obtained serum until later for further studies [47]. Analysis of tryptase concentration during anaphylaxis compared to its baseline level (> $24 \mathrm{~h}$ since resolution of the symptoms) is currently the most recommended and widely available marker of anaphylaxis. The increased concentration of tryptase that remains within the reference range $(0-11.4 \mu \mathrm{g} / \mathrm{l})$, but exceeds the value calculated with the formula $(1.2 \times$ baseline serum tryptase concentration +2 ), confirms activation of the mastocytes during the hypersensitivity reaction [48].

\section{Treatment and prevention}

Adrenaline remains the principal and the first-line treatment recommended intervention in anaphylaxis [12]. Its rapid and timely administration is critical to adequately manage severe symptoms and to avoid a biphasic reaction [49]. When applied within 30 min from the onset of the first symptoms, it decreases the risk of biphasic reaction by 23\% [50]. Undiluted solution 1: 1000 $(1 \mathrm{mg} / \mathrm{ml})$ of adrenaline is administered intramuscularly at a dose of $0.01 \mathrm{mg} / \mathrm{kg}$ (maximum dose $0.5 \mathrm{mg}$ in adults, and $0.3 \mathrm{mg}$ in children) in the anterolateral side of the thigh [1]. Regardless of the site [51], it is recommended to deliver adrenaline intramuscularly instead of subcutaneously since the former resulted in a higher plasma concentration peak (2136 \pm 351 vs. $1802 \pm 214$ pg/ml) and faster onset of action ( $8 \pm 2$ vs. $34 \pm 14 \mathrm{~min}$ ) [52]. When needed, adrenaline injections are repeated every 5 to 15 min usually within initial $2 \mathrm{~h}$ following the first dose [5]. Therefore a 4-hour long observation is optimal for monitoring children with anaphylaxis [53]. In the medical settings, the first adrenaline dose is followed by fast infusion of the intravenous fluids such as $0.9 \%$ sodium chloride at $10-20 \mathrm{ml} / \mathrm{kg}$ over $10 \mathrm{~min}$ in case of hypotension unresponsive to intramuscular adrenaline, oxygen supplementation, comfort position of the child with the lower extremities elevated for effective cardiac output. The upright position during anaphylaxis is associated with a greater fatality risk resulting from the empty right ventricle syndrome [54]. Antihistamines and glucocorticosteroids (GCS) may be considered in anaphylaxis management only as the second-line medi- 
cations [55]. The previous studies failed to demonstrate their ability to prevent biphasic reactions. In fact, the latest data indicated that systemic GCS in anaphylaxis might even increase the risk of biphasic reaction in children [49]. The antihistamines are justified only as medications against bothersome symptoms of urticaria and pruritus. It is crucial that use of either antihistamines or GCS does not delay adrenaline administration in anaphylaxis. In the latest European systematic literature review of 50 studies that included a total of 18,449 participants, adrenaline was verified to be the only disease-modifying intervention in anaphylaxis [56]. Unfortunately, both medical personnel and parents hesitate to use adrenaline as the primary intervention in anaphylaxis in children despite their prior training and data showing a reduced risk of hospitalization, including the risk of stay in the ICU after pre-hospital adrenaline injection [57, 58]. Introduction of new modified autoinjectors might improve the above statistics because they can facilitate appropriate adrenaline administration. Similarly, face-to-face training regardless of its duration may improve knowledge about management in anaphylaxis. There is also some beneficial effect of electronic learning modules [59] and multidisciplinary education programs [60]. Autoinjectors, currently available in Europe include 3 adrenaline doses: 0.15 mg (EpiPen Junior, Emerade, Altellus, Anapen Junior), 0.3 mg (EpiPen Senior, Emerade, Altellus, Anapen Senior) and $0.5 \mathrm{mg}$ (Emerade). In Poland, the following are available: EpiPen 0.15 and $0.30 \mathrm{mg}$ and a pre-filled syringe with adrenaline WZF $0.15 \mathrm{mg}$ (registered, but not yet available in the market) and $0.3 \mathrm{mg}$. In the USA, in addition to EpiPen autoinjectors, there are also Adrenaclick and Auvi-Q. In 2020, Auvi-Q was the first one in the world to introduce a nominal dose of $0.1 \mathrm{mg}$ dedicated for children with a body weight of 7.5-15 kg [61]. According to EMA and EAACl guidelines, all the patients with a risk of anaphylaxis are recommended to always have two packs of adrenaline, especially during the COVID-19 pandemic $[62,63]$.

The secondary prevention, in addition to prevention of exposure to the anaphylaxis trigger, includes allergen immunotherapy (Hymenoptera venom), and desensitization to certain medications (acetylsalicylic acid, chemotherapeutic agents, monoclonal antibodies, antibiotics, hormones) according to predefined protocols when indicated for essential therapy $[13,38]$.

The options of prevention to food-induced anaphylaxis were further expanded in January 2020 when the FDA approved Aimmune's Palforzia, a peanut allergen in powder form for oral immunotherapy (OIT). The medication is indicated for the patients aged 7-14 years with a clinically confirmed allergy to peanuts. There is a pending FDA decision regarding Viaskin Peanut for epicutaneous immunotherapy for the same indications as above. The results of phase III clinical trials of that medication are very encouraging [64].
The way to promote immunotolerance to various food allergens is their use in thermally processed form, such as cooking or baking for milk, baking or pasteurization for eggs [65]. Desensitization in allergy to wheat flour is carried out with a ready-made commercial formulation $[66,67]$, while treatment in red meat allergy should start with $1 \%$ diluted boiled beef extract and eventually cooked beef [68]. There are numerous algorithms to help with a gradual dose increase of the above products. Low-dose OIT is to promote tolerance to the amount that may occur due to accidental food contamination. It corresponds to $1 / 32$ of hen's egg, $3 \mathrm{ml}$ of cow's milk, 50-75 mg of wheat protein and 125-300 mg of peanut protein, which is safe and useful in the management of children with food-induced anaphylaxis. The good age to start OIT would be approximately 5 years [69].

\section{Conclusions}

The current epidemiological data, despite apparent differences in incidence and prevalence, indicate an increase in anaphylaxis cases especially in the developed countries. The upcoming ICD-11 classification will enable more accurate reporting of cases and probably improve the quality of population data. At the same time, simplifying anaphylaxis criteria should facilitate its diagnosis in daily practice, hence enable faster administration of adrenaline, the first-line intervention in anaphylaxis. Unfortunately, adrenaline is still not used in children as often as needed by both medical personnel and parents. Identification of the potential causative factor is now possible thanks to the modern component-resolved diagnostics, which starts to play a major role in the secondary prophylaxis. Such prophylaxis is based not only on the prevention of contacts with the noxious allergen, but also development of broadly-defined specific immunotolerance, which should significantly improve quality of life of the patient who had recovered from the anaphylactic shock in the past.

\section{Conflict of interest}

The authors declare no conflict of interest.

\section{References}

1. Sampson HA, Munoz-Furlong A, Campbell RL, et al. Second symposium on the definition and management of anaphylaxis: summary report -Second National Institute of Allergy and Infectious Disease/Food Allergy and Anaphylaxis Network symposium. J Allergy Clin Immunol 2006; 117: 391-7.

2. Brown SG, Mullins RJ, Gold MS. Anaphylaxis: diagnosis and management. Med J Aust 2006; 185: 283-9.

3. Panesar SS, Javad S, de Silva D, et al.; EAACI Food Allergy and Anaphylaxis Group. The epidemiology of anaphylaxis in Europe: a systematic review. Allergy 2013; 68: 1353-61.

4. Lieberman P, Camargo CA, Bohlke K, et al. Epidemiology of anaphylaxis: findings of the American College of Allergy, 
Asthma and Immunology Epidemiology of Anaphylaxis Working Group. Ann Allergy Asthma Immunol 2006; 97: 596-602.

5. Simons FER, Ardusso LRF, Bilò MB, et al. World allergy organization guidelines for the assessment and management of anaphylaxis. World Allergy Organ I 2011; 4: 13-37.

6. Muraro A, Roberts G, Worm M, et al. Anaphylaxis: guidelines from the European Academy of Allergy and Clinical Immunology. Allergy 2014; 69: 1026-45.

7. Loprinzi Brauer CE, Motosue MS, Li JT, et al. Prospective validation of the NIAID/FAAN criteria for emergency department diagnosis of anaphylaxis. J Allergy Clin Immunol Pract 2016; 4: 1220-6.

8. Turner PJ, Worm M, Ansotegui IJ, et al. WAO Anaphylaxis Committee. Time to revisit the definition and clinical criteria for anaphylaxis? World Allergy Organ J 2019; 12: 100066.

9. Tanno LK, Bierrenbach AL, Simons FER, et al. Critical view of anaphylaxis epidemiology: open questions and new perspectives. Allergy Asthma Clin Immunol 2018; 14: 12.

10. Turner PJ, Campbell DE, Motosue MS, Campbell RL. Global Trends in Anaphylaxis Epidemiology and Clinical Implications. J Allergy Clin Immunol Pract 2020; 8: 1169-76.

11. Worm M, Moneret-Vautrin A, Scherer K, et al. First European data from the network of severe allergic reactions (NORA). Allergy 2014; 69: 1397-404.

12. Grabenhenrich LB, Dölle S, Ruëff F, et al. Epinephrine in severe allergic reactions: the European Anaphylaxis Register. J Allergy Clin Immunol Pract 2018; 6: 1898-906.

13. Francuzik W, Ruëff F, Bauer A, et al. Phenotype and risk factors of venom-induced anaphylaxis: a case-control study of the European Anaphylaxis Registry. J Allergy Clin Immunol 2020. DOI: 10.1016/j.jaci.2020.06.008.

14. Grabenhenrich LB, Dölle S, Moneret-Vautrin A, et al. Anaphylaxis in children and adolescents: the European Anaphylaxis Registry. J Allergy Clin Immunol 2016; 137: 1128-37.

15. Aurich S, Dölle-Bierke S, Francuzik W, et al. Anaphylaxis in elderly patients-data from the European Anaphylaxis Registry. Front Immunol 2019; 10: 750.

16. Kraft M, Knop MP, Renaudin JM, et al. Network for Online Registration of Anaphylaxis (NORA). Secondary prevention measures in anaphylaxis patients: data from the anaphylaxis registry. Allergy 2020; 75: 901-10.

17. Asai Y, Yanishevsky Y, Clarke A, et al. Rate, triggers, severity and management of anaphylaxis in adults treated in a Canadian emergency department. Int Arch Allergy Immunol 2014; 164: 246-52.

18. Mullins RJ, Wainstein BK, Barnes EH, et al. Increases in anaphylaxis fatalities in Australia from 1997 to 2013. Clin Exp Allergy 2016; 46: 1099-110.

19. Jahnz-Rozyk K, Raciborski F, Śliwczyński AM, et al. Anaphylaxis in Poland: the epidemiology and direct costs. Adv Dermatol Allergol 2017; 34: 573-9.

20. Wang Y, Allen KJ, Suaini NHA, et al. The global incidence and prevalence of anaphylaxis in children in the general population: a systematic review. Allergy 2019; 74: 1063-80.

21. Pouessel G, Turner PJ, Worm M, et al. Food-induced fatal anaphylaxis: from epidemiological data to general prevention strategies. Clin Exp Allergy 2018; 48: 1584-93.

22. Turner PJ, Baumert J, Beyer K, et al. Can we identify patients at risk of lifethreatening allergic reactions to food? Allergy 2016; 71: 1241-55.

23. Francuzik W, Nassiri M, Babina M, Worm M. Impact of sex on anaphylaxis severity-data from the Anaphylaxis Registry. J Allergy Clin Immunol 2015; 136: 1425-6.
24. Pouessel G, Tanno LK, Claverie C, et al. Fatal anaphylaxis in children in France: Analysis of national data. Pediatr Allergy Immunol 2018; 29: 101-4.

25. Turner PJ, Jerschow E, Umasunthar T, et al. Fatal anaphylaxis: mortality rate and risk factors. J Allergy Clin Immunol Pract 2017; 5: 1169-78.

26. Kim SY, Kim MH, Cho YJ. Different clinical features of anaphylaxis according to cause and risk factors for severe reactions. Allergol Int 2018; 67: 96-102.

27. Robinson LB, Arroyo AC, Faridi MK, et al. Trends in US hospitalizations for anaphylaxis among infants and toddlers: 2006 to 2015. Ann Allergy Asthma Immunol 2021; 126: 168174.e3.

28. Rudders SA, Arias SA, Camargo CA Jr. Trends in hospitalizations for food-induced anaphylaxis in US children, 20002009. J Allergy Clin Immunol 2014; 134: 960-2.e3.

29. Nocerino R, Leone L, Cosenza L, Berni Canani R. Increasing rate of hospitalizations for food-induced anaphylaxis in Italian children: an analysis of the Italian Ministry of Health database. J Allergy Clin Immunol 2015; 135: 833-5.

30. Mullins RJ, Dear KB, Tang ML. Time trends in Australian hospital anaphylaxis admissions in 1998-1999 to 2011-2012. J Allergy Clin Immunol 2015; 136: 367-75.

31. Simons FE. Anaphylaxis. J Allergy Clin Immunol 2010; 125 (2 Suppl 2): 161-81.

32. Cheng BT, Fishbein AB. Patterns of hospital readmission in children with food-induced anaphylaxis. J Allergy Clin Immunol Pract 2020; 8: 1420-2.e1.

33. Wright CD, Longjohn M, Lieberman PL, Lieberman JA. An analysis of anaphylaxis cases at a single pediatric emergency department during a 1-year period. Ann Allergy Asthma Immunol 2017; 118: 461-4.

34. Skypala IJ. Food-induced anaphylaxis: role of hidden allergens and cofactors. Front Immunol 2019; 10: 673.

35. Heaps A, Carter S, Selwood C, et al. The utility of the ISAC allergen array in the investigation of idiopathic anaphylaxis. Clin Exp Immunol 2014; 177: 483-90.

36. Chong KW, Ruiz-Garcia M, Patel N, et al. Reaction phenotypes in IgE-mediated food allergy and anaphylaxis. Ann Allergy Asthma Immunol 2020; 124: 473-8.

37. Tarczoń I, Jedynak-Wąsowicz U, Lis G, et al. Is the clinical manifestation of anaphylaxis in children influenced by the trigger of reaction? Adv Dermatol Allergol 2021; 38: 788-97.

38. Xing Y, Zhang H, Sun S, et al. Clinical features and treatment of pediatric patients with drug-induced anaphylaxis: a study based on pharmacovigilance data. Eur J Pediatr 2018; 177: 145-54.

39. Khaleva E, Franz A, Garvey LH, et al. Perioperative anaphylaxis in children: etiology, time sequence, and patterns of clinical reactivity. Pediatr Allergy Immunol 2020; 31: 85-94.

40. Hertzog JH, Preisberga K, Penfil S. The incidence and nature of allergic and anaphylactic reactions during pediatric procedural sedation: a report from the Pediatric Sedation Research Consortium. Hosp Pediatr 2019; 9: 16-23.

41. Muller YD, Vionnet J, Beyeler F, et al. Management of allergy transfer upon solid organ transplantation. Am J Transplant 2020; 20: 834-43.

42. Olabarri M, Vazquez P, Gonzalez-Posada A, et al. Risk factors for severe anaphylaxis in children. J Pediatr 2020; 225: 193-7.

43. Dribin TE, Michelson KA, Zhang Y, et al. Are children with a history of asthma more likely to have severe anaphylactic reactions? A retrospective cohort study. J Pediatr 2020; 220: 159-64. 
44. Beck SC, Wilding T, Buka RJ, et al. Biomarkers in human anaphylaxis: a critical appraisal of current evidence and perspectives. Front Immunol 2019; 10: 494.

45. Sun KJ, He JT, Huang HY, et al. Diagnostic role of serum tryptase in anaphylactic deaths in forensic medicine: a systematic review and meta-analysis. Forensic Sci Med Pathol 2018; 14: 209-15.

46. Buka RJ, Knibb RC, Crossman RJ, et al. Anaphylaxis and clinical utility of real-world measurement of acute serum tryptase in UK emergency departments. J Allergy Clin Immunol Pract 2017; 5: 1280-7.

47. Platzgummer S, Bizzaro N, Bilò MB, et al. Recommendations for the use of tryptase in the diagnosis of anaphylaxis and clonal mastcell disorders. Eur Ann Allergy Clin Immunol 2020; 52: 51-61.

48. Baretto RL, Beck S, Heslegrave J, et al. Validation of international consensus equation for acute serum total tryptase in mast cell activation: a perioperative perspective. Allergy 2017; 72: 2031-4.

49. Shaker MS, Wallace DV, Golden DBK, et al. Anaphylaxisa 2020 practice parameter update, systematic review, and Grading of Recommendations, Assessment, Development and Evaluation (GRADE) analysis. J Allergy Clin Immunol 2020; 145: 1082-123.

50. Liu X, Lee S, Lohse CM, et al. Biphasic reactions in emergency department anaphylaxis patients: a prospective cohort study. J Allergy Clin Immunol Pract 2020; 8: 1230-8.

51. Wang Q, Pittman L, Healey A, et al. Ideal body position for epinephrine autoinjector administration. Allergy Asthma Proc 2021; 42: 147-52.

52. Simons FE, Roberts JR, Gu X, Simons KJ. Epinephrine absorption in children with a history of anaphylaxis. J Allergy Clin Immunol 1998; 101: 33-7.

53. Dribin TE, Michelson KA, Monuteaux MC, et al. Timing and predictors of repeat epinephrine administration among children hospitalized for anaphylaxis. J Allergy Clin Immunol Pract 2020; 8: 1400-2.

54. McLure M, Eastwood K, Parr M, Bray J. A rapid review of advanced life support guidelines for cardiac arrest associated with anaphylaxis. Resuscitation 2021; 159: 137-49.

55. Campbell RL, Li JT, Nicklas RA, Sadosty AT. Emergency department diagnosis and treatment of anaphylaxis: a practice parameter. Ann Allergy Asthma Immunol 2014; 113: 599-608.

56. De Silva D, Singh C, Muraro A, et al. Diagnosing, managing and preventing anaphylaxis: systematic review. Allergy 2021; 76: 1493-506.

57. Tarczoń I, Jedynak-Wąsowicz U, Lis G, et al. Intervention in anaphylaxis: the experience of one paediatric centre based on NORA reports. Adv Dermatol Allergol 2021; 38: 235-43.

58. Trainor JL, Pittsenbarger ZE, Joshi D, et al. Outcomes and factors associated with prehospital treatment of pediatric anaphylaxis. Pediatr Emerg Care 2022; 38: e69-74.

59. Rasmussen E, Goddard AG, Bayer DK. Use of electronic learning modules can improve medical trainee knowledge regarding anaphylaxis diagnosis and treatment. Ann Allergy Asthma Immunol 2020; 1243: 295-6.

60. Polloni L, Baldi I, Lazzarotto F, et al. Multidisciplinary education improves school personnel's self-efficacy in managing food allergy and anaphylaxis. Pediatr Allergy Immunol 2020; 31: 380-7.

61. Brown JC, Simons E, Rudders SA. Epinephrine in the management of anaphylaxis. J Allergy Clin Immunol Pract 2020; 8: 1186-95.
62. Kraft M, Dölle-Bierke S, Turner PJ, et al. EAACI Task force Clinical epidemiology of anaphylaxis: experts' perspective on the use of adrenaline autoinjectors in Europe. Clin Transl Allergy 2020; 10: 12.

63. Casale TB, Wang J, Nowak-Węgrzyn A. Acute at home management of anaphylaxis during the Covid-19 pandemic. J Allergy Clin Immunol Pract 2020; 8: 1795-7.

64. Wang J. Advances in the management of peanut allergy (oral immunotherapy and epicutaneous immunotherapy). Allergy Asthma Proc 2020; 41: 5-9.

65. Gruzelle V, Juchet A, Martin-Blondel A, et al. Benefits of baked milk oral immunotherapy in French children with cow's milk allergy. Pediatr Allergy Immunol 2020; 31: 364-70.

66. Nowak-Węgrzyn A, Wood RA, Nadeau KC, et al. Multicenter, randomized, double-blind, placebo-controlled clinical trial of vital wheat gluten oral immunotherapy. J Allergy Clin Immunol 2019; 143: 651-61.

67. Nagakura KI, Yanagida N, Sato S, et al. Low-dose-oral immunotherapy for children with wheat-induced anaphylaxis. Pediatr Allergy Immunol 2020; 31: 371-9.

68. Yucel E, Sipahi Cimen S, Varol S, et al. Red meat desensitization in a child with delayed anaphylaxis due to alpha-Gal allergy. Pediatr Allergy Immunol 2019; 30: 771-3.

69. Nagakura KI, Sato S, Asaumi T, et al. Novel insights regarding anaphylaxis in children - with a focus on prevalence, diagnosis, and treatment. Pediatr Allergy Immunol 2020; 31: 879-88. 